\title{
ENGENHEIROS NA COZINHA: O PROGRAMA MASTERCHEF COMO INSPIRAÇÃO PARA ESTUDOS PRÁTICOS DE TEMPOS E MOVIMENTOS
}

\author{
Leonardo Menezes Melo - leonardommelo@hotmail.com \\ Centro Universitário Estácio de Sá SC - Departamento de Engenharia de Produção \\ Av. Leoberto Leal,431 - Barreiros \\ 88.110-001 - São José - Santa Catarina
}

\begin{abstract}
Resumo: O processo de ensino-aprendizagem presente nas universidades precisa estar em constante metamorfose, visto que as tecnologias, as necessidades da sociedade e as partes envolvidas no meio acadêmico se alteram com o passar dos anos. No decorrer dos últimos anos, universidades e professores, cada vez mais utilizam metodologia ativa e suas técnicas no processo de ensino-aprendizagem, contribuindo com alunos mais ativos nos encontros $e$ intensificando a relação de teoria e prática. Especificamente para o processo de ensinoaprendizagem nas Engenharias, torna-se necessário e relevante a aquisição de tal conhecimento e a aplicação junto as partes envolvidas. Este trabalho tem o objetivo de relatar as dinâmicas e os resultados obtidos durante o processo ensino-aprendizagem na disciplina de Engenharia de Métodos, do curso de Engenharia de Produção, por meio da inspiração no programa MasterChef com o uso da técnica intitulado problem-based learning. As atividades aconteceram no primeiro semestre de 2019, sendo planejadas e executadas por meio da reflexão e debate dos conceitos e técnicas existentes no estudo de tempos e movimentos, e com aplicações em receitas desenvolvidas e gravadas pelos próprios alunos. Como principais resultados tem-se: participação intensiva dos alunos, relação acentuada entre teoria e prática, percepção por parte dos envolvidos que o conteúdo e toda a dinâmica jamais serão esquecidos, desenvolvimento/aprimoramento de habilidades culinárias, e, elevado grau de satisfação dos alunos com o desenvolvimento das atividades.
\end{abstract}

Palavras-chave: Engenharia de métodos. Tempos e movimentos. Metodologia ativa. Problem-based learning. MasterChef.

\section{INTRODUÇÃO}

As mudanças constantes presentes no mundo fazem com que os seres racionais necessitem ficar atentos aos diversos cenários que se apresentam com o passar do tempo. Tais situações ocorrem em qualquer local, desde por meio do desenvolvimento de uma nova tecnologia até a maneira na qual o ser humano se relaciona com outros indivíduos em sociedade.

No que tange ao processo de ensino-aprendizagem, as mudanças também são constantes, novos produtos tecnológicos são disponibilizados e utilizados pelos professores para debates e exposição de pensamentos (e.g. quadro e giz ou projetor e computador), bem como novas dinâmicas são necessárias para a interação entre as partes envolvidas (professores e alunos) visto que são pessoas de gerações diferentes, e, por muitos momentos, com expectativas distintas. 
A metodologia ativa contribui significativamente com o ensino no que se refere o repensar das práticas e das ações docentes perante a uma sociedade em constante mudança, principalmente pelo fato de ser um "método institucional, aplicado em sala de aula, onde os alunos participam do processo de aprendizagem. Os alunos executam atividades significativas e pensam sobre o que estão fazendo", conforme mencionado por Prince (2004) apud Venturini e Silva (2018, p. 61).

Diversas são as técnicas existentes e relacionadas com a metodologia ativa, que podem ser utilizados pelos docentes no decorrer do processo de ensino-aprendizagem, alinhados ao cenário apresentado, como: gamification, project based learning, problem-based learning, problematização $e$ think-pair-share.

As práticas docentes utilizadas, no que tange a formação de futuros engenheiros, necessitam principalmente por meio dos professores, do Núcleo Docente Estruturante (NDE) e do colegiado, estarem constantemente atentos aos novos cenários e possibilidades, que venham a realizar contribuições significativas na formação dos futuros engenheiros, preparando profissionais mais qualificados e prontos para os desafios da profissão. O interesse ao conteúdo debatido, a compreensão/reflexão e a inserção de metodologias ativas contribuem com tal cenário.

Com base no mencionado, os alunos do curso de Engenharia de Produção do Centro Universitário Estácio de Sá de Santa Catarina, turma da disciplina de Engenharia de Métodos do primeiro semestre de 2019, foram desafiados a participar e desenvolver atividades durante o decorrer de todo o semestre da dinâmica intitulada "MasterChef: Engenharia de Métodos", que visava contribuir intensamente na relação entre teoria e prática, bem como na maneira ativa de se aprender.

A inspiração da prática foi no programa de culinária intitulado "MasterChef Brasil" que tem como dinâmica a seleção e participação de cozinheiros, que são desafiados a criar e(ou) reproduzir receitas em um intervalo de tempo, com os recursos disponibilizados para avaliação dos jurados. No final da temporada existe formalmente apenas um único vencedor. O programa é "[...]produzido pela Endemol Shine Brasil em uma coprodução com a Band e o Discovery Home \& Health" (REDAÇÃO, 2020).

O objetivo deste trabalho é relatar como aconteceram as dinâmicas e quais foram os resultados obtidos no decorrer do processo de ensino-aprendizagem da disciplina de Engenharia de Métodos, por meio da inspiração no programa MasterChef.

A relação entre a disciplina e o programa ocorreu pelo fato dos assuntos abordados relacionarem-se com as análises de tempos e movimentos, com o intuito de propiciar a experiência na disciplina de maneira mais intensa, com práticas e com elevada aproximação no que se referem aos estudos de tempos e movimentos em demandas similares presentes no mercado de trabalho e na vida dos envolvidos, contribuindo com a formação de engenheiros ainda mais qualificados.

\section{REFERÊNCIAL TEÓRICO}

Neste referencial teórico tem-se a contextualização para os leitores sobre a metodologia ativa no que tange ao método denominado problem-based learning (PBL), e sobre os estudos de tempos e movimentos presentes na disciplina denominada Engenharia de Métodos.

\subsection{Metodologia ativa e o problem-based learning}

Participação, engajamento, autogestão do aprendizado, autonomia, e reflexão são algumas das palavras-chave relacionadas as metodologias ativas (VENTURINI E SILVA, 
2018). Para tal, o aluno torna-se o elemento central e que necessita ter relação intensiva com as palavras mencionadas, principalmente pelo estímulo e incentivo dos docentes.

No que tange especificamente a reflexão, a metodologia ativa é um ...

método fundamentado em atividades que solicitam que o aluno faça algo, e, ao mesmo tempo, reflita sobre isso. O professor figura um orientador, não sendo a única fonte de conhecimento do processo. "A diferença fundamental que caracteriza um ambiente de aprendizagem ativa é a atitude ativa da inteligência”. (BARBOSA E MOURA, 2014 apud VENTURINI E SILVA, 2018, p.64)

As atividades propostas necessitam ser desafiadoras, provocantes e tentadoras, tendo o viés de estimular aos alunos a despertar a curiosidade e o espírito de competitividade, no que tange aos outros grupos e(ou) ao próprio sujeito, a buscar a melhor solução para a situação.

Gamification, project based learning, problem-based learning, problematização $e$ thinkpair-share são alguns dos métodos existentes nos estudos sobre metodologia ativa, que necessitam ser compreendidos pelos professores, para conhecimento de opções e seleção daquele com potencial para ser utilizado na disciplina a ser ministrada, conforme situação proposta e alinhamento com o conteúdo debatido.

Destaca-se que o problem-based learning, conhecido e debatido também apenas por meio da abreviação PBL, é o método geralmente mais utilizado na Engenharia de Produção, de acordo com o levantamento realizado por Santos e Figueiredo (2018) ao avaliar os anais do Encontro Nacional de Engenharia de Produção (ENEGEP) de 2013 a 2017. O PBL “[...] é uma metodologia de ensino e aprendizagem que utiliza problemas - coerentes para uma futura atuação dos alunos como profissionais e cidadão[...]" (RIBEIRO, 2008, p. 24).

Com a utilização do PBL em determinadas disciplinas, os alunos se deparam com situações ou simulações de cenários da vida real, buscando motivar e envolver a turma no que tocante a relação de teoria e prática em vivências cotidianas, tanto em atividades rotineiras presentes na vida do ser humano quanto em situações que os engenheiros venham a se deparar ao exercer as atividades profissionais.

Dentre as atividades exercidas no mercado de trabalho pelos engenheiros, os problemas são aqueles que geralmente se apresentam de maneira mais constante, desde os classificados como simples aos complexos. Com isso, a possibilidade dos alunos de vivenciarem problemas similares em sala de aula, faz com que os mesmos estejam cada vez mais preparados para situações que serão vivenciadas durante as atividades exercidas na profissão, bem como, sendo um importante motivador no que tange o desenvolvimento das atividades por parte dos alunos.

\subsection{Contextualizando a engenharia de métodos}

Engenharia de Métodos é uma disciplina clássica presente nos cursos de Engenharia de Produção, com estudos e discussões referentes aos processos produtivos, no que tange tempos e movimentos existentes nas execuções das atividades, possuindo relação direta com a gestão, bem como, sendo apresentada e inicialmente desenvolvida por autores clássicos e consagrados.

Os termos "Engenharia de Métodos", "Projeto de Trabalho", "Estudo de Trabalho" e "Projeto de Métodos" são sinônimos atualmente consolidados e por muitas vezes mais presentes e utilizados em substituição ao "estudo de tempo e movimento", conforme visualizado e informado previamente por Ralph Mosser Barnes (importante pesquisador do tema) no livro clássico denominado "Estudo de movimentos e tempos: Projeto e medida do trabalho", de 1977. Cardoso (2018, p. 17) ao mencionar que a "Engenharia de Métodos é um 
dos termos utilizados em substituição a Estudo de Movimentos e de Tempo", enfatiza a terminologia que atualmente é frequentemente mais utilizada. De maneira geral, a Engenharia de Métodos refere-se ao estudo dos processos, no que tange aos tempos empregados e movimentos executados durante o desenvolvimento das atividades. Barnes (1977, p.1) descreve que

\begin{abstract}
o estudo de movimentos e de tempos é o estudo sistemático dos sistemas de trabalho com os seguintes objetivos: (1) desenvolver o sistema e o método preferido, usualmente aquele de menor custo; (2) padronizar esse sistema e método; (3) determinar o tempo gasto por uma pessoa qualificada e devidamente treinada, trabalhando num ritmo normal, para executar uma tarefa ou operação específica; e (4) orientar o treinamento do trabalhador no método preferido.
\end{abstract}

O estudo de tempos foi introduzido em 1881 por Frederick Winslow Taylor, e o estudo de movimentos pelo casal Frank Bunker Gilbreth e Lilian Moller Gilbreth com o intuito de diminuir ou eliminar movimentos considerados desnecessários durante a execução das atividades (TOLEDO JUNIOR, 1985).

Nos estudos de tempos e movimentos existem diversas técnicas e métodos disponíveis para que os processos sejam descritos e avaliados sistematicamente. Dentre essas, tem-se o "Fluxograma" que é "[...]uma técnica para se registrar um processo de maneira compacta, a fim de tornar possível sua melhor compreensão e posterior melhoria" (BARNES, 1977, p. 46), e o "Mapofluxograma" que de maneira geral é a união do mapa local com o processo, sendo "outra representação gráfica que auxilia o profissional a estabelecer a sequência dos processos dentro das dependências da organização[...]" (SELEME, 2009, p. 50).

O "Diagrama Homem x Máquina", outro método disponível, representa de maneira combinada as ações de trabalho independente, combinado e de espera entre o homem e a(s) máquina(s) durante a execução do processo analisado, sendo também registrados os tempos empregados (SELEME, 2009).

Já o "Gráfico Simo" representa os movimentos do corpo humano durante a execução das atividades. Torna-se importante ressaltar que o casal Gilbreth propôs o estudo dos micros movimentos, dividindo em dezessete movimentos fundamentais da mão, para avaliação das ações executadas no decorrer das atividades.

A folha de "Análise de Operações" auxilia de maneira sistemática no que se refere a busca por melhorias para o processo, contribuindo por exemplo com a redução de estoques, encurtamento das distâncias, pesquisa por novas ferramentas ou dispositivos, avaliação de preparação das máquinas, qualificação dos operadores e avaliação das condições de trabalho. Para tal, existem questões-chave desenvolvidas e fragmentadas em seis grupos: materiais; manuseio de materiais; ferramentas, dispositivos e gabaritos; máquinas; operador; e, condições de trabalho (BARNES, 1977).

A "Avaliação do Ritmo" existe devido necessidade de estabelecer os tempos das atividades, para isso, necessita-se avaliar o ritmo do operador-padrão (SELEME, 2009). O operador-padrão também contribui para avaliação do ritmo dos outros operadores, podendo estabelecer os mais ágeis e mais lentos. Dentre os diversos métodos de avaliação do ritmo tem-se o de repartir 52 cartas em quatro pilhas em um quadrado de $25 \mathrm{~cm}$ (BARNES, 1977).

O "Estudo de Tempo" é utilizado para determinação do tempo-padrão da atividade executada por uma pessoa qualificada e bem treinada. De maneira geral, existem as necessidades de: registro do tempo, determinação do tamanho da amostra a serem cronometrados, avaliação do ritmo do operador, determinação de tolerâncias existentes e necessárias (e.g. ida ao banheiro etc.), e por fim o cálculo do tempo-padrão (BARNES, 1977). 


\section{METODOLOGIA}

Este trabalho relaciona-se a Pesquisa-ação que é ...

[...]um tipo de pesquisa com base empírica que é concebida e realizada em estreita associação com uma ação ou com a resolução de um problema coletivo e no qual os pesquisadores e participantes representativos da situação ou do problema estão envolvidos de modo cooperativo ou participativo. (Thiollent, 1985, p. 14 apud Gil, 2002, p.55)

Para tal, as situações-problema (cenários para análises durante todo o semestre) foram estabelecidas pelos próprios alunos, com o intuito de relacionar e aproximar a teoria debatida com a prática, no que tange situações que os alunos possam se deparar no mercado de trabalho.

Inicialmente tornou-se necessário apresentar a disciplina e alguns assuntos, com o intuito de melhor compreensão das temáticas e atividades a serem desenvolvidas durante os encontros programados para a disciplina. Em seguida, realizou-se a etapa de "1-Apresentação do Desafio" intitulado "MasterChef: Engenharia de Métodos" e ocorreu a "2-Divisão dos grupos".

O decorrer do semestre e as atividades planejadas ocorreram sequencialmente da etapa 3 até a 10 (ver Figura 1), sendo apresentadas na seção seguinte deste trabalho. Torna-se importante ressaltar que a "Filmagem" (etapa 3) serve de referência para a execução das próximas etapas, necessitando de atenção e concentração especial. Erros nessa etapa tendem a comprometer todas as seguintes.

Figura 1 - Etapas desenvolvidas durante o decorrer do semestre e relacionadas ao desafio MasterChef: Engenharia de Métodos

\begin{tabular}{|c|c|}
\hline 1 & •Apresentação do Desafio \\
\hline 2 & •Divisão dos Grupos \\
\hline 3 & •Filmagem \\
\hline 4 & •Fluxograma \\
\hline 5 & •Mapofluxograma \\
\hline 6 & •Diagrama Homem x Máquina \\
\hline 7 & •Gráfico Simo \\
\hline 8 & •Análise de Operações \\
\hline 9 & •Avaliação do Ritmo \\
\hline 10 & •Estudo de Tempo \\
\hline
\end{tabular}

Fonte: Próprio autor

\section{RESULTADOS}

A etapa inicial ("1 - Apresentação do Desafio") ocorreu por meio da exposição aos envolvidos sobre a proposta de trabalho. O desafio foi apresentado com o título de "MasterChef: Engenharia de Métodos", sendo necessário contextualizar a dinâmica do programa de televisão, visto que alguns (poucos) alunos não conheciam o programa. A 
primeira reação dos alunos foi de espanto com uma mistura de surpresa, provavelmente pelo fato de ser necessário "ir para a cozinha", visto que a maioria da turma é formada por alunos do sexo masculino, e a própria sociedade, geralmente com costumes tradicionais, pouco incentiva a participação de pessoas do sexo masculino na realização de atividades na cozinha.

A “2 - Divisão dos Grupos” foi realizada pelos próprios alunos, conforme afinidades e proximidades existentes entre os participantes. De maneira geral, os grupos possuíam em média quatro participantes, que ficaram com a tarefa de avaliar e escolher a receita de trabalho, bem como mencionar e validar a escolha no próximo encontro com o professor. As receitas foram das mais diversas, conforme é possível observar no Quadro 1. Apenas um grupo escolheu a preparação de bebidas, sendo aprovado, visto que o objetivo principal estava na realização das filmagens para posterior análise e desenvolvimento das habilidades nos conhecimentos oriundos pela disciplina.

Quadro 1 - Receitas selecionadas pelos grupos

\begin{tabular}{|c|l|}
\hline Grupos & Receitas \\
\hline 1 & Bebida (Passion Tonica) \\
\hline 2 & Churrasco: Assar carne e linguiça em churrasqueira \\
\hline 3 & Preparação de massa de panquecas \\
\hline 4 & Preparação de Salada \\
\hline 5 & Massa de macarrão \\
\hline
\end{tabular}

Fonte: Próprio autor

Para a etapa de "3 - Filmagem" foram avaliadas e debatidas algumas recomendações teóricas baseada em livros indicados para a disciplina, como posição das câmeras, iluminação adequada e momento adequado para a filmagem. Os principais requisitos para as filmagens foram: utilizar pelo menos duas câmeras ao mesmo tempo, uma filmando a bancada de trabalho e outra o local com uma visão mais geral. A primeira tinha como objetivo principal avaliar as movimentações das mãos sobre a bancada de trabalho, e a segunda, avaliar a movimentação do cozinheiro. Era preciso realizar a gravação sem pausas, para que fosse possível conhecer os tempos para a realização das tarefas, bem como, criar um vídeo editado para que o mesmo pudesse ser assistido por todos da turma no encontro em sala.

Com base no conhecimento detalhado e filmado durante as preparações das receitas, foram desenvolvidos o "4 - Fluxograma" e o "5 - Mapofluxograma". Na Figura 2 tem-se o exemplo do fluxograma desenvolvido pelo grupo 4 durante a preparação de salada, com a utilização das simbologias e da apresentação dos tempos e das distâncias percorridas em cada atividade descrita pelo grupo. Com fluxograma criado, torna-se possível, em um primeiro momento, avaliar que na maioria das etapas ocorre a operação, com pouco transporte, inspeção e armazenamento, e, sem a existência de espera, aparentemente sendo um processo com pouco desperdício.

Já na Figura 3 tem-se como exemplo o mapofluxograma desenvolvido pelo grupo 3, ao realizar o estudo da preparação de massa de panquecas. Percebe-se que o colaborador, ao preparar a massa, necessita se deslocar a um ponto e posteriormente voltar a alguma região já visitada, situação observada entre as atividades "11-12" e "13-23", ou mesmo entre "24-31" e "32”, sendo locais de possíveis melhorias no processo.

Por consequência da natureza da atividade, a criação dos mapofluxogramas fez com que os grupos fossem desafiados a encontrar e utilizar alguma ferramenta para desenho da planta baixa do local de trabalho, sendo um conhecimento transcendente aos pertinentes a disciplina. O mapofluxograma contribui com a percepção do espaço e do movimento no ambiente, sendo possível avaliar e buscar reduzir/eliminar o cruzamento das linhas do fluxo. 
Figura 2 - Fluxograma desenvolvido pelo grupo 4

(Preparação de salada) com a orientação do professor

\begin{tabular}{|c|c|c|c|c|c|c|c|c|c|c|}
\hline \multirow{2}{*}{\multicolumn{5}{|c|}{ Categorias }} & \multicolumn{2}{|c|}{ Atual } & \multicolumn{2}{|c|}{ Proposto } & Diferenças & enças \\
\hline & & & & & № & Tempd & № & Tempo & № & Tempo \\
\hline & & \multicolumn{3}{|c|}{ Operação } & 7 & $8 \mathrm{~min}$ & & & & \\
\hline & & \multicolumn{3}{|c|}{ Transporte } & 2 & $1 \mathrm{~min}$ & & & & \\
\hline & & \multicolumn{3}{|c|}{ Inspeção } & 2 & $1 \mathrm{~min}$ & & & & \\
\hline & & \multicolumn{3}{|l|}{ Espera } & - & - & & & & \\
\hline & & \multicolumn{3}{|c|}{ Armazenagem } & 1 & $1 \mathrm{~min}$ & & & & \\
\hline & \multicolumn{4}{|c|}{ Distância Percorrida } & $3 \mathrm{mts}$ & $11 \mathrm{~min}$ & & & & \\
\hline & \multirow[b]{2}{*}{ 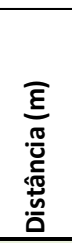 } & \multirow[b]{2}{*}{ 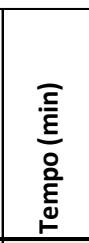 } & \multicolumn{5}{|c|}{ Categoria } & \multirow{2}{*}{\multicolumn{3}{|c|}{ Descrição }} \\
\hline & & & 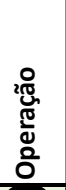 & 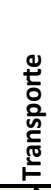 & 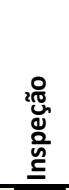 & 劳 & 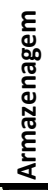 & & & \\
\hline \multirow{5}{*}{ 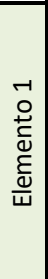 } & 0,5 & 0,50 & & & & & & \multicolumn{3}{|c|}{ Selecionar os equipamentos } \\
\hline & 0,5 & 0,50 & & & & & & \multicolumn{3}{|c|}{ Organizar os equipamentos } \\
\hline & 0,5 & 0,50 & & & & & & \multicolumn{3}{|c|}{ Selecionar os materiais (legumes) } \\
\hline & 0,5 & 0,50 & & & & & & \multicolumn{3}{|c|}{ Organizar os materiais (legumes) } \\
\hline & 0 & 0,17 & & & & & & \multicolumn{3}{|c|}{ Lavar as mãos } \\
\hline \multirow{5}{*}{ 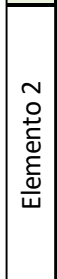 } & 0 & 2,00 & & & & & & \multicolumn{3}{|c|}{ Lavar os materiais (legumes) } \\
\hline & 0,5 & 4,50 & & & & & & \multicolumn{3}{|c|}{ Cortar os materiais } \\
\hline & 0,5 & 0,50 & & & & & & \multicolumn{3}{|c|}{ Selecionar os materiais (descarte) } \\
\hline & 0 & 0,33 & & & & & & \multicolumn{3}{|c|}{ Abrir lata (material - grão de bico) } \\
\hline & 0 & 1,00 & & & & & & \multicolumn{3}{|c|}{ Montar a salada } \\
\hline \multirow{2}{*}{\begin{tabular}{|c|}
$m$ \\
$\dot{\varepsilon}$ \\
$\frac{\omega}{\omega}$ \\
\end{tabular}} & 0 & 0,25 & & & & & & \multicolumn{3}{|c|}{ Inserir chia na salada pronta (à gosto) } \\
\hline & 0 & 0,25 & & E & & ) & & \multicolumn{3}{|c|}{ Inserir azeite na salada pronta (à gosto) } \\
\hline
\end{tabular}

Fonte: Próprio autor

Figura 3 - Mapofluxograma desenvolvido pelo grupo 3

(Preparação de massa de panquecas) com a orientação do professor

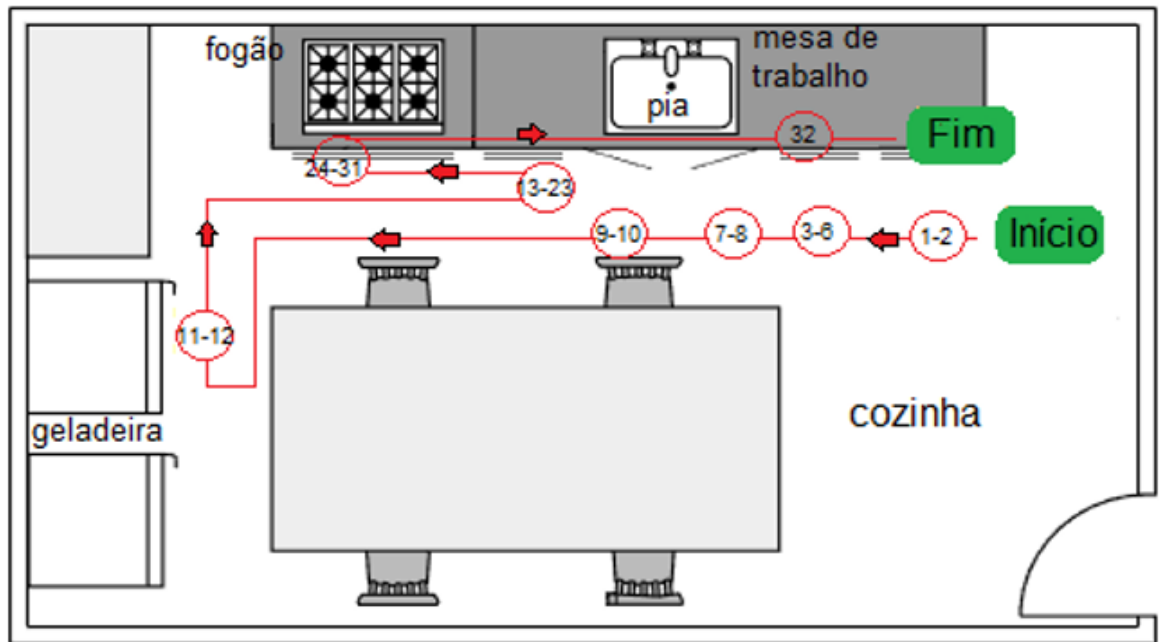

Fonte: Próprio autor 
O “6 - Diagrama Homem x Máquina” permite visualizar individualmente e comparar o percentual de operação efetiva do homem e da máquina, possibilitando avaliar situações de ganho, principalmente no que tange a redução dos tempos e melhoria da eficiência. $\mathrm{O}$ grupo 3 desenvolveu o diagrama e resumiu o percentual de atividades independentes, combinadas e de espera entre o homem e a máquina (Quadro 2). Posteriormente foram realizadas melhorias no processo para otimização e por consequência alteração dos tempos.

Quadro 2 - Resumo do Diagrama Homem x Máquina desenvolvido pelo grupo 3

(Preparação de massa de panquecas) com a orientação do professor

\begin{tabular}{|l|l|c|c|c|c|}
\hline \multicolumn{6}{|c|}{ Diagrama de atividades múltiplas para preparação das panquecas } \\
\hline Processo: preparar panquecas & \multicolumn{2}{|c|}{ Momem } & \multicolumn{2}{c|}{ Máquinas } \\
\hline & & \multicolumn{2}{|c|}{ Hempo (s) } & $\%$ & Tempo (s) \\
\cline { 2 - 6 } & & $\%$ & 13 & 56 & 150 \\
\cline { 2 - 6 } & Atividades independentes & 5 & 102 & 39 & 102 \\
\cline { 2 - 6 } & Atividades combinadas & 39 & 150 & 5 & 13 \\
\cline { 2 - 6 } & Espera & 56 & 265 & & 265 \\
\cline { 2 - 6 } & totais & & & & \\
\hline
\end{tabular}

Fonte: Próprio autor

O "7 - Gráfico Simo" apresentado pelos grupos relata no decorrer do tempo todas as ações realizadas pela mão esquerda e pela mão direita. Ao avaliar os casos dos grupos, percebeu-se que em alguns a mão direita tem uma sobrecarga de trabalho, estando quase todo o tempo em ação. Tal avaliação torna-se relevante, pelo fato de buscar desenvolver/melhorar o processo, no intuito de dividir as atividades executadas pelas mãos, por consequência, minimizando o afastamento de trabalhadores devido sobrecarga em uma das mãos.

$\mathrm{Na}$ "8 - Análise de Operações" cada grupo avaliou e respondeu os seis grupos de perguntas propostos na teoria. A situação auxiliou na reflexão sobre o cenário atual e na busca por melhorias. É um momento desafiador, que exige concentração e ao mesmo tempo criatividade dos participantes, sendo uma das etapas mais difíceis. Como exemplo tem-se o grupo 3, que ao responder uma das perguntas no tópico denominado "ferramentas, dispositivos e gabaritos" concluiu que a utilização de uma frigideira antiaderente eliminaria o uso de óleo.

A "9 - Avaliação do Ritmo" foi realizada em sala de aula, principalmente junto aos alunos que foram os responsáveis pela preparação das receitas. Utilizou-se o método de repartir o baralho em quatro pilhas dentro de um quadro de aproximadamente $25 \mathrm{~cm}$ de lado (visualizar a Figura 4). Um aluno realizava a separação e outro cronometrava e anotava os tempos. Posteriormente os tempos foram avaliados e comparados com padrões internacionais, para avaliação do ritmo do operador-padrão.

Figura 4 - Alunos realizando a avaliação do ritmo com o método de repartir o baralho

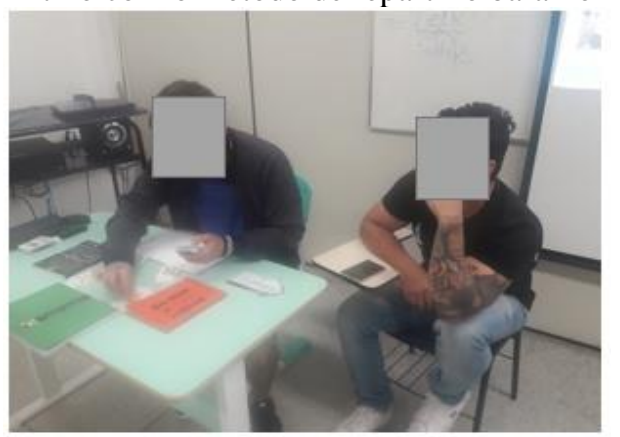

Fonte: Próprio autor 
No "10 - Estudo do Tempo" torna-se necessário realizar alguns exercícios em paralelo às atividades do desafio MasterChef, principalmente por conta da existência de cálculos. Posteriormente os alunos realizaram os cálculos e as avaliações para cada receita. $\mathrm{O}$ momento é relevante pelo fato de existir reflexão e cálculos sobre quantidade de cronometragens para se atingir a confiabilidade desejada, a necessidade de avaliar o ritmo do operador, definição das tolerâncias existentes no ambiente de trabalho, e, finalmente, determinar o tempo-padrão. Como exemplo, o grupo 1 (Bebida - Passion Tonica), fragmentou o processo em elementos (conjunto de atividades que se relacionam), realizou os cálculos de amostragem, e, por meio dos tempos cronometrados com média de 10,5 minutos, calculou o tempo normal (que considera o ritmo do operador) resultando em 12,075 minutos e o tempo-padrão (que considera as tolerâncias) resultando em 13,42 minutos.

\section{CONSIDERAÇÕES FINAIS}

A possibilidade de literalmente "colocar a mão na massa", situação ocorrida com os alunos participantes da disciplina de Engenharia de Métodos, permitiu vivências intensas e que para os envolvidos tornaram-se inesquecíveis, tanto por meio da filmagem como de toda a dinâmica.

A participação e entrega durante o desenvolvimento das atividades, de maneira geral, ocorreu conforme esperado e de maneira gratificante, gerando a sensação de dever cumprido e de que os objetivos propostos foram alcançados, principalmente no que tange a relação de teoria e prática por meio da aproximação das vivências acadêmicas com aquelas existentes no mercado de trabalho.

Desde a apresentação do desafio até o estudo do tempo, a entrega dos alunos foi constante e satisfatória, propiciada pela implementação da dinâmica na disciplina. Os exemplos dos resultados obtidos pelos alunos e contidos na seção de "Resultados" deste trabalho corroboram com tal afirmativa.

Para finalizar, destaca-se que, posteriormente a finalização da disciplina, uma aluna relatou com um tom de gratidão e compartilhamento, que estava vivenciando na prática (na empresa que trabalha) o estudo de tempos e movimentos, e, em diversos momentos, lembrava de situações debatidas em sala e realizadas durante o desafio MasterChef: Engenharia de Métodos.

\section{REFERÊNCIAS}

BARNES, Ralph Mosser. Estudo de movimentos e tempos: projeto e medida do trabalho. 6 . ed. São Paulo: Edgard Blucher, 1977. 635 p.

CARDOSO, Wagner. Engenharia de Métodos e Produtividade. Ananindeua: Itacaiúnas, 2018. 148 p.

GIL, Antonio Carlos. Como elaborar projetos de pesquisa. 4. ed. São Paulo: Atlas, 2002. $175 \mathrm{p}$.

REDAÇÃO, da. Inscrições para sétima temporada estão abertas. 2020. Disponível em: $<$ https://entretenimento.band.uol.com.br/masterchef/noticias/100000981368/inscricoes-parasetima-temporada-do-masterchef-brasil-estao-abertas.html>. Acesso em: 25 jan. 2020. 
"Os desafios para formar hoje o engenheiro do amanhã"

RIBEIRO, Luis Roberto de Camargo. Aprendizagem baseada em problemas (pbl) na educação em engenharia. Revista de Ensino de Engenharia, [s. L.], v. 27, n. 2, p.23-32, 2008.

SANTOS, Carolina Maia dos; FIGUEIREDO, Fernanda Abreu de Moraes. Estudos sobre metodologias de aprendizagem ativa na engenharia publicados nos anais do enegep de 2013 a 2017. In: XXXVIII ENCONTRO NACIONAL DE ENGENHARIA DE PRODUCAO, 1., 2018, Manaus. [s. L.]: Abepro, 2018. p. 1 - 16.

SELEME, Robson. Métodos e tempos: racionalizando a produção de bens e serviços. Curitiba: Ibpex, 2009. 160 p.

TOLEDO JUNIOR, Itys-fides Bueno de. Tempos \& Métodos. Mogi das Cruzes: O\&m Assessoria, Escola e Editora, 1985. 181 p.

VENTURINI, Simone Ferigolo; SILVA, Taís Oliveira. Uso e benefícios das metodologias ativas em uma disciplina de engenharia de produção. Cippus, Canoas, v. 6, n. 1, p.59-74, 2018.

\title{
ENGINEERS IN THE KITCHEN: THE MASTERCHEF PROGRAM AS INSPIRATION FOR PRACTICAL ON TIME-MOTION STUDIES
}

\begin{abstract}
The teaching-learning process present in universities needs to be in constant metamorphosis, since the technologies, the needs of society and the parties involved in the academic environment are changing over the years. Over the past few years, universities and professors have increasingly used active learning and its techniques in the teaching-learning process, contributing to more active students in the meetings and intensifying the relationship between theory and practice. Specifically for the teaching-learning process in Engineering, it is necessary and relevant to acquire such knowledge and apply it to the parties involved. This work aims to report the dynamics and results obtained during the teaching-learning process in the discipline of Methods Engineering, of the Production Engineering course, through inspiration in the MasterChef program with the use of the technique called problem-based learning. The activities took place in the first semester of 2019, being planned and executed through reflection and debate of the concepts and techniques existing in the study of times and motions, and with applications in recipes developed and recorded by the students themselves. The main results are: intensive students participation, strong relationship between theory and practice, perception by those involved that the content and all the dynamics will never be forgotten, development / improvement of culinary skills, and a high degree of student satisfaction with the development of activities.
\end{abstract}

Keywords: Methods Engineering. Times and motions. Active learning. Problem-based learning. MasterChef. 